\title{
Variational Nodal Transport Methods for Hexagonal and Three-Dimensional Geometries
}

\author{
Final Report
}

Elmer E. Lewis

Department of Mechanical Engineering McCormick School of Engineering and Applied Science Northwestern University

Evanston, IL 60208

February, 1992

This report was preparec as an account of work sponsored by the United States Government. Neither the United States nor the Department of Energy, nor any of their employees, nor any of their contractors, subcontractors, or their employees, makes any warranty, express or implied, or assumes any legal liability or responsibility for the accuracy, completeness, or usefulness of any information, apparatus, product or process disclosed or represents that its use would not infringe privately-owned rights.

Prepared for

The U. S. Department of Energy

Agreement No. DE-FG07-88ER 12810 


\begin{abstract}
The properties of the variational nodal method for neutron transport calculations are investigated. The method is generalized for three-dimensional multigroup criticality problems in both hexagonal-z and Cartesian geometries. The method is implemented as part of the Argonne National Laboratory Code DIF3D, and applied to a series of benchmark reactor calculations. Variational nodal methods are compared of nodal transport methods based on both interface-current and discrete ordinate approximations. Model problems are used to examine the effect of running each of the three classes of nodal transport methods on computers with massively parallel architectures.
\end{abstract}

Introduction: In this investigation the variational nodal method for neutron transport calculations has been formulated in three-dimensional hexagonal and Cartesian geometries. It has been implemented as nodules of the Argonne National Laboratory production code DIF3D in a form suitable for multigroup reactor calculations. Ten publications resulted entirely or in part from work performed under this contract. They are listed as references (1) through (10) ard are included as appendices with the same numbers. Appendix (10) gives the most comprehensive description of the work. The doctoral dissertations of two Northwestern University students are also the result of this investigation.

Background: At the initiation of this contract, the principal investigator and his students had formulated the variational nodal method for the numerical solution of neutron transport problems. Studies had been made of the analytic and numerical properties of the variational approach. At that time, however, the method had been incorporated only into a stand-alone code treating fixed-source, one-energy-group problems in $x-y$ geometry. Thus the code was capable only of treating model problems which were of academic interest, but was of little direct value to zeactor designers. Moreover, the solution algorithm indicated that the method was far from competitive with other nodal transport methods on a accuracy per CPU cost basis.

Accomplishments: The most important accomplishment of this project has been to take the variational nodal method from the academic 
curriosity described above to a working tool for reactor designers to use in doing three-dimensional multigroup neutron transport criticality calculations. Implementations of variational nodal methods are now incorporated into the DIF3D production code at Argonne National Laboratory. Other than Monte Carlo, this code is at present the only transport method available in the United States for doing threedimensional reactor calculation in hexagonal geometry. Moreover, for those geometries for which other methods exist, the three-dimensional Takeda benchmark problems indicate that the variational nodal method is more accurate than the existing ANL interface current transport nodal method, and frequently comparable to fine-mesh $S_{8}$ calculations. 10

Staff members of the Reactor Analysis Division at ANL now are using the variational nodal code for Hex-z calculations associated with the Experimental Breeder Reactor II. The code is presently being modified for use in a work station environment by ANL staff, with the intention of using it for routine EBR II analysis. Generalization of the variational nodal method to include both within-group and group-to-group anisotropic scattering was initiated during the last year of the contract. Implementation has been completed in the two-dimensional geometries. It is expected that within a few months the incorporation of anisotropic scattering into the three-dimensional versions of the DIF3D variational nodal modules will be completed.

The research in variational nodal methods required to achieve these accomplishment was centered around two tasks. First, the generation of the large arrays of integrals was automated through the use of symbolic manipulation. This process, discussed in some detail in section II of Appendix (10), was necessary to eliminate the intractable task of analytically evaluating thousands of two and three-dimensional integrals by hand. Second, a partitioned red-black solution algorithm was formulated and developed. As discussed in Section III of Appendix (10), this algorithm greatly reduced the computing times involved in variational nodal nodal calculations in part though making improved use of the vector architecture of the Cray X-MP on which the codes are routinely used.

During the course of the contract, the investigation was widened to compare variational nodal methods to the other two classes of nodal transport methods and to examine the effects of emerging massively parallel computer architectures on nodal transport calculations. We have been able to demonstrate that nodal discrete ordinate as well as the existing ANL interface-current method and the variational nodal method 
may be formulated as response matrices and solved using red-black algorithms. Discrete ordinate formulations, beginning with diamonddifferencing 9 and extended to the nodal linear-linear approximation 8 offer interesting points for comparing the nodal methods. We are presently running one-group $x-y$ version of each of these methods on the Connection Machines-2, -200 and -5 in order to study the effects of massively parallel architectures on nodal solution algorithm. Comparisons of accuracy of variational nodal and discrete ordinate methods have been made, ${ }^{3}$ It the near future it should be possible to compare all three classes of nodal methods as $x-y$ options of the Code DIF3D on a vector supercomputer, on work stations, and on massively parallel computers.

Finally, near the end of the contract period we discovered a reduced set of angular trial functions 7 which reduces the size of the response matrices and thus the running times substantially without a commensurate loss of accuracy. We have thus far only investigated these trial for $x-y$ geometry model problems, but they may have significant potential in three dimensions and/or as operators for synthetic acceleration of higher angular order approximations.

Personnel: In addition to the the Principal investigator, there were two students supported under this contract. C. M. Carrico, completed his $\mathrm{PhD}$. in the fall of 1991 and joined the staff of the Reactor Analysis Division of Argonne National Laboratory. U. Hanebutte is currently completing his $\mathrm{PhD}$ dissertation at Northwestern.

ANL Collaboration: Progress toward the objectives of this contract were greatly facilitated by in interest and support provided by the Reactor Analysis Division of Argonne National Laboratory. In addition to providing the computing facilities for the project and summer employment for C. B. Carrico to work on the project at ANL, a visiting staff member, P. Palmiotti, was assigned to the work. This allowed its scope could be expanded to include anisotropic scattering and the formulation of methods in all of the three-dimensional geometries, i. e. $x$ $y-z$, triangular-z and hex-z. 


\section{Publications:}

(1) U. R. Hanebutte \& E. E. Lewis, " A Discrete Ordinate Response Matrix Method for Massively Parallel Computers," Proc. Int. Mtg Adv. in Mathematics. computations, and Reactor Physics. Pittsburgh, PA, April 28 - May 1, 1991, Vol. 1, 1.1 1-1,11, Am. Nucl. Soc. 1991

(2) C. B. Carrico \& E. E. Lewis, "Variational Nodal Solution Algorithms for Multigroup Criticality Problems," Proc. Int. Mtg. Adv_ in Mathematics computations, and Reactor Physics. Pittsburgh, PA, April 28 - May 1, 1991, Vol. 2, 7.1 4-1,10, Am. Nucl. Soc. 1991

(3) U. R. Hanebutte, C. B. Carrico \& E. E. Lewis, "Comparison of Sn and Pn Response Matrix Methods for Massively Parallel Computers,"Trans. Am. Nucl, Soc. 63. 181-182 (1991)

(4) C. B. Carrico,E. E. Lewis \& G. Palmiotti, "Nodal Variational Transport Methods for Triangular and Hexagonal Geometries," Trans. Am. Nucl. Soc. 63, 186-187 (1991)

(5) C. B. Carrico, G. Palmiotti \& E. E. Lewis, "Variational Nodal Methods in Three Dimensions: Cartesian, Triangular and Hexagonal Geometries" Trans. Am. Nucl. Soc. 63.173-174 (1991)

(6) U. R. Hanebutte \& E. E. Lewis, "A Generalized Discrete Ordinate Response Matrix Formulation for Anisotropic Scattering," Trans. Am. Nucl. Soc. 63.178-179(1991)

(7) C. B. Carrico, E. E. Lewis \& G. Palmiotti, "A Reduced Trial Function Set for the Variational Nodal Methods" Trans. Am. Nucl. Soc. 64. (1992) submitted

(8) U. R. Hanebutte \& E. E. Lewis, "A Massively Parallel Nodal Discrete Ordinate Method," Trans. Am. Nucl. Sos. 64.(1992) submitted

(9) U. R. Hanebutte \& E. E. Lewis, " A Massively Parallel Discrete Ordinate Response Matrix Method for Neutron Transport," Nucl. Sci. Eng. accepted for publication

(10) C. B. Carrico, E. E. Lewis \& G. Palmiotti, " Three-Dimensional Variational Nodal Transport Methods for Cartesian, Triangular and Hexagonal Criticality Calculations," Nucl. Sci. Eng, accepted for publication

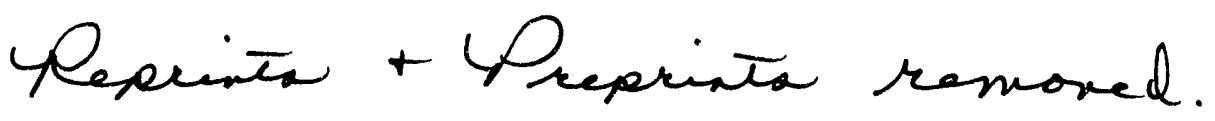



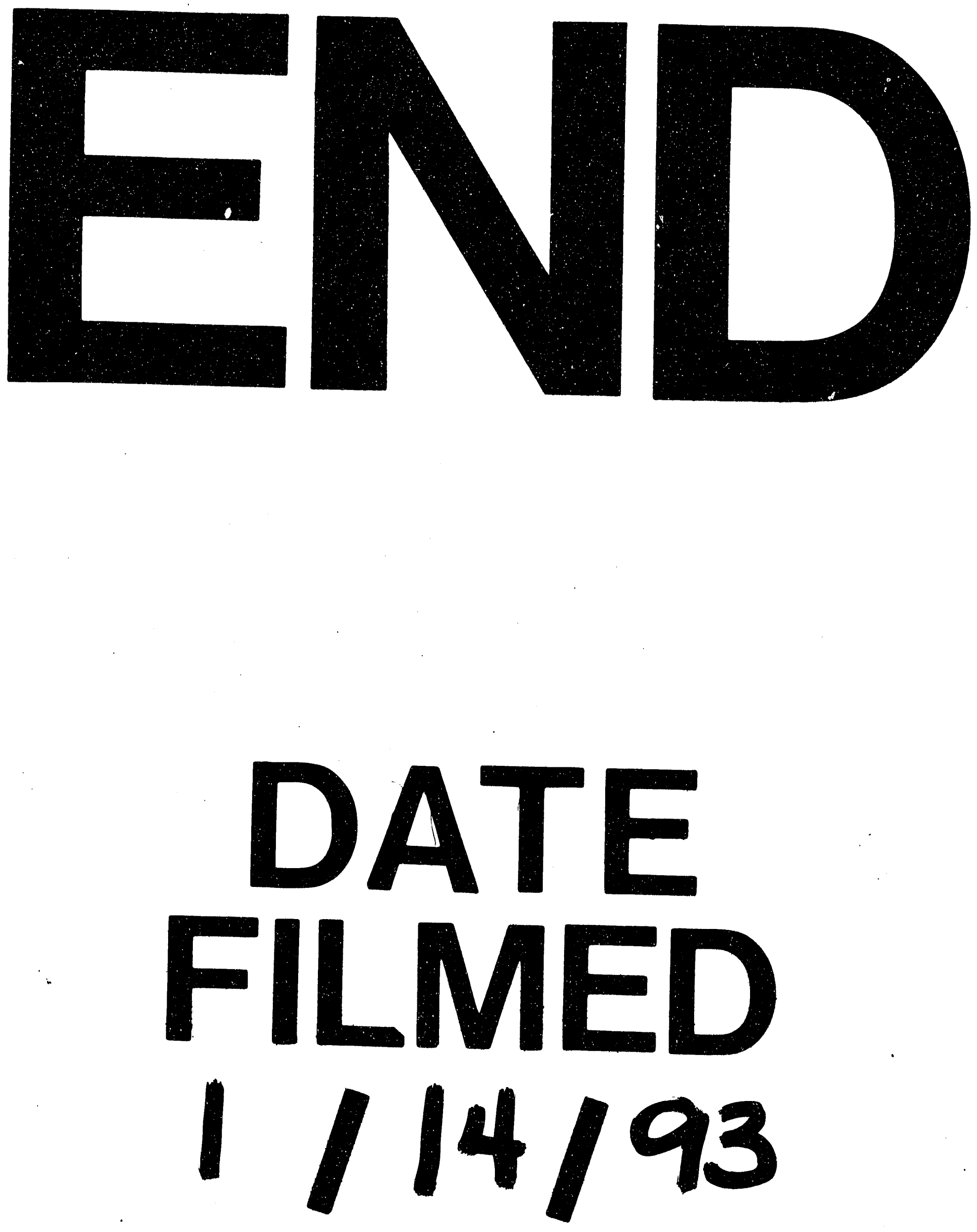
